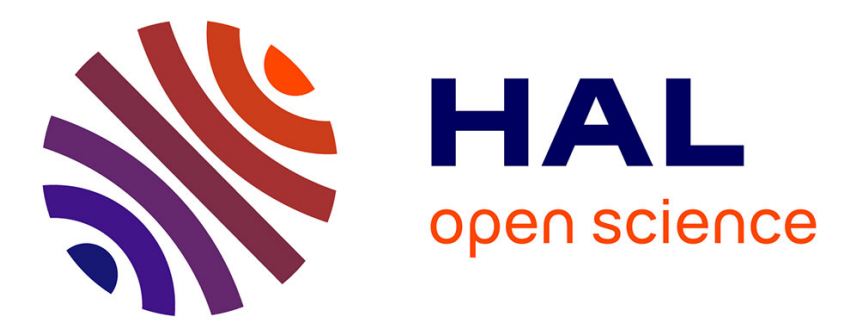

\title{
Brain-Machine Collaboration for Cyborg Intelligence
}

\author{
Zhongzhi Shi, Gang Ma, Shu Wang, Jianqing Li
}

\section{To cite this version:}

Zhongzhi Shi, Gang Ma, Shu Wang, Jianqing Li. Brain-Machine Collaboration for Cyborg Intelligence. 9th International Conference on Intelligent Information Processing (IIP), Nov 2016, Melbourne, VIC, Australia. pp.256-266, 10.1007/978-3-319-48390-0_26 . hal-01615002

\section{HAL Id: hal-01615002 \\ https://hal.inria.fr/hal-01615002}

Submitted on 11 Oct 2017

HAL is a multi-disciplinary open access archive for the deposit and dissemination of scientific research documents, whether they are published or not. The documents may come from teaching and research institutions in France or abroad, or from public or private research centers.
L'archive ouverte pluridisciplinaire HAL, est destinée au dépôt et à la diffusion de documents scientifiques de niveau recherche, publiés ou non, émanant des établissements d'enseignement et de recherche français ou étrangers, des laboratoires publics ou privés. 


\title{
Brain-Machine Collaboration for Cyborg Intelligence
}

\author{
Zhongzhi Shi ${ }^{1}$, Gang $\mathrm{Ma}^{1,2}$, Shu Wang, ${ }^{1,2}$, Jianqing $\mathrm{Li}^{1,2}$ \\ 1 Key Laboratory of Intelligent Information Processing \\ Institute of Computing Technology, Chinese Academy of Sciences \\ Beijing 100190, China \\ 2 University of Chinese Academy of Sciences, Beijing 100049, China \\ \{shizz, mag,wangs,lijq\}@ics.ict.ac.cn
}

\begin{abstract}
Cyborg intelligence integrates the best of both machine and biological intelligences via brain-machine integration. To make this integration effective and co-adaptive biological brain and machine should work collaboratively. Both environment awareness based collaboration and motivation based collaboration will be presented in the paper. Motivation is the cause of action and plays important roles in collaboration. The motivation leaning method and algorithm will be explored in terms of event curiosity, which is useful for sharing common interest situations.
\end{abstract}

Keywords: cyborg intelligence, brain-machine collaboration, motivation driven collaboration, motivation learning, mind model CAM

\section{Introduction}

Cyborg intelligence aims to integrate AI with biological intelligence by closely and deeply connecting computer and biological beings. The term cyborg was presented by Clynes and Kline in 1960 [1], to describe a being with both organic and computing components. Combined with electronic sensing and navigation technology, a guided rat can be developed into an effective 'robot' that will possess several natural advantages over current mobile robots [2]. Supported by the National Program on Key Basic Research Project we are engaging in the research on Computational Theory and Method of Perception and Cognition of Brain-Machine Integration. The main goal is the exploration of cyborg intelligence through brain-machine integration, enhancing strengths and compensating for weaknesses by combining the biological cognition capability with the computer computational capability. To make this integration effective and co-adaptive, multi-agents should work collaboratively on environment perception, information processing, and command execution.

Collaborations occur over time as organizations interact formally and informally through repetitive sequences of negotiation, development of commitments, and execution of those commitments. Both cooperation and coordination may occur as part of the early process of collaboration, collaboration represents a longer-term integrated process. Gray describes collaboration as "a process through which parties who see different aspects of a problem can constructively explore their differences and search for solutions that go beyond their own limited vision of what is possible." [3]

In multi-agent system no single agent owns all knowledge required for solving complex tasks. Agents work together to achieve common goals, which are beyond the 
capabilities of individual agent. Each agent perceives information from the environment with sensors and find out the number of cognitive tasks, even same task of having different information and the possible combination of tasks for execution for certain interval of time, pick out or select the particular combination for execution in an interval of time, and finally outputs the required effective actions to the environment.

As an internal mental model of agent, BDI model has been well recognized in philosophical and artificial intelligence area. Bratman's philosophical theory was formalized by Cohen and Levesque [4] and other researchers. A cognitive model for multi-agent collaboration should consider external perception and internal mental state of agents. Awareness is knowledge created through interaction between an agent and its environment. In multi-agent system group awareness is an understanding of the activities of others and provides a context for own activity. Group awareness can be divided into basic questions about who is collaborating, what they are doing, and where they are working. Gutwin etc. proposed a conceptual framework of workspace awareness that structures thinking about groupware interface support. They list elements for the conceptual framework [5]. Workspace awareness in a particular situation is made up of some combination of these elements.

Collaboration is goal-oriented and aided by motivation. Psychologists define motivation as an internal process that activates, guides, and maintains behavior over time. Mook defined motivation as "the cause of action" briefly [6]. Maslow proposed hierarchy of needs which was one of first unified motivation theories [7]. MicroPsi concerns modeling a motivational system to solve in the pursuit of a given set of goals, which reflects cognitive, social and physiological needs, and can account for individual variance and personality traits [8].

In this paper, a collaborative agent model for cyborg intelligence will be proposed in terms of external environment awareness and internal mental state. The agent intention is driven by motivation which is generated dynamically.

\section{Cyborg Intelligence}

Cyborg intelligence is dedicated to integrating AI with biological intelligence by tightly connecting machines and biological beings, for example, via brain-machine interfaces (BMIs) [9]. Figure 1 shows the physical implementation of the rat-robot navigation system [10]. In the automatic navigation of rats, five bipolar stimulating electrodes separately are implanted in medial forebrain bundle (MFB), somatosensory cortices (SI), and periaqueductal gray matter (PAG) of the rat brain. There is also a backpack fixed on the rat to receive the wireless commands.

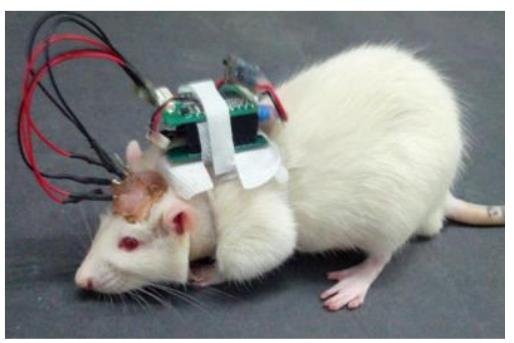

Fig. 1. Rat cyborg

There are two components which are necessary to implement the automatic 
navigation. Firstly, the communication between a computer and a rat needs to be solved. The stimulation signals are delivered by a wireless backpack stimulator which is comprised of stimulating circuit, control processor and Bluetooth transceivers. The control processor receives the computer instructions through the Bluetooth transceivers. Then it sends commands to the stimulator to control the rat behaviors. By receiving commands from the machine, the rat can perform a lot of navigation tasks, e.g. walking around mazes, climbing bridges, and stopping at a special place. Secondly, a video camera device used to capture the rat movement is installed above the scenario. With the video captured by the birdeye camera, the machine can establish a map of the environment and analyze the real time kinetic state of the rat.

In brain-machine integration, each rat brain and computer can be viewed as an agent playing special role and work together for a sharing goal. The agent cognitive model is illustrated in Figure 2, which agents deliberate the external perception and the internal mental state for decision-making. The model is represented as a 4-tuple: 〈Awareness, Belief, Goal, Plan $\rangle$. Awareness is described by the basic elements and relationships related to the agent's setting. Belief can be viewed as the agent's knowledge about its environment and itself. Goal represents the concrete motivations that influence an agent's behaviors. Plan is used to achieve the agent's goals. Moreover, an important module motivation-driven intention is used to drive the collaboration of cyborg intelligent system.

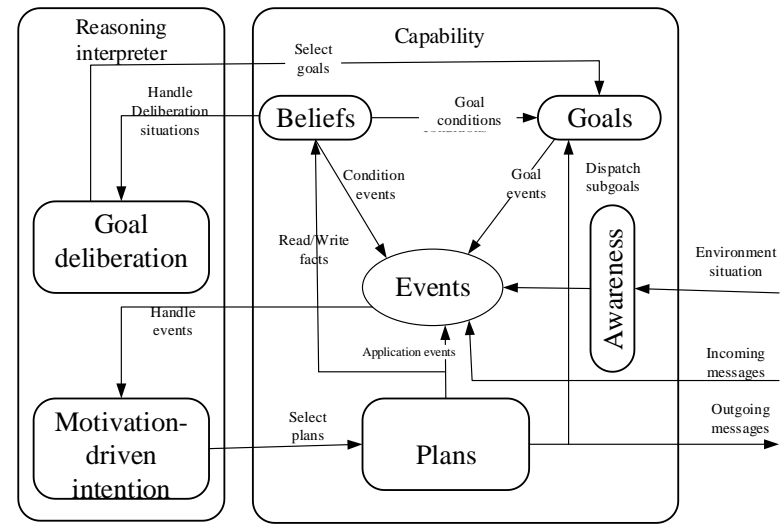

Fig. 2. Agent cognitive model

\section{Environment Awareness}

The environment is the complex combination of physical conditions and agents responses. Cyborg intelligent systems require bidirectional information perception between rat brain and computer. Awareness is the state or ability to perceive, to feel events, objects or sensory patterns, and cognitive reaction to a condition or event. Awareness has four basic characteristics:

- Awareness is knowledge about the state of a particular environment.

- Environments change over time, so awareness must be kept up to date.

- Agents maintain their awareness by interacting with the environment.

- Awareness establishes usually an event. 
The brain computer collaborative awareness model is defined as 2-tuples: \{Element, Relation \}, where Element of awareness is described as follows:

a) Who: describes the existence of agent and identity the role, answer question who is participating?

b) What: shows agent's actions and abilities, answer question what are they doing? And what can they do? Also can show intentions to answer question what are they going to do?

c) Where: indicates the location of agents, answer question where are they?

d) When: shows the time point of agent behavior, answer question when can action execute?

Basic relationships contain task relationship, role relationship, operation relationship, activity relationship and cooperation relationships.

a) Task relationships define task decomposition and composition relationships. Task involves activities with a clear and unique role attribute

b) Role relationships describe the role relationship of agents in the multi-agent activities.

c) Operation relationships describe the operation set of agent.

d) Activity relationships describe activity of the role at a time.

e) Cooperation relationships describe the interactions between agents. A partnership can be investigated through cooperation activities relevance between agents to ensure the transmission of information between different perception of the role and tasks for maintenance of the entire multi-agent perception.

Based on the integration of Marr visual theory and Gestalt whole perception theory, applying statistical learning and deep learning and other methods to analyze visual information of environment and generate high-level semantics. The convolutional generative stochastic model (CGSM) is proposed for the visual awareness [11]. The Generative Stochastic Networks (GSN) is based on learning the transition operator of a Markov chain whose stationary distribution estimates the data distribution. GSN has the capability to obtain a generative model of the data distribution without explicitly specifying a probabilistic graphical model, and allows learning deep generative model through global training via back-propagation. In order to seek a visual awareness path with a stronger robustness and a better hierarchy feature representation with gradually more global representation in the higher levels, the CGSM model can be stacked through multi convolutional generative stochastic layers. The mean-pooling is applied to CGSM so as to the downward pass operation can be successfully implemented. The layer-wise sampling like deep Boltzmann machine network will be adopted in the computational graph. CGSM has a strong robustness for noisy data, and is better to serve as a visual awareness pathway.

\section{Motivation Driven Collaboration}

Motivation is an internal motive force and subjective reasons, which direct drive the individual activities to achieve a certain purpose, and the psychological state initiated and maintained by individual activities. Psychologists define motivation as the process that initiates, guides, and maintains goal-oriented behaviors. All kinds of behaviors and activities of the people can't be separated from the motivation. Motivation has the following functions: 
a) Arouse the start function of the action. Personally, all the power of his actions must be through his mind, must be changed to his desire for motivation, in order to make him act up.

b) A directing function that focuses an individual's behavior towards or away from specific goals.

c) An organizing function that influences the combination of behavioral components into coherent, goal-oriented behavioral sequences.

d) Strengthen function of motivation. One's experience of successes and failures on the activity, have certain influence on his activity ambition. In other words, how is the behavioral result, influencing people's motivation? Therefore the motivation plays a regulation control appearing in the form of positive or negative reinforcement role in people's behavior.

Consider the dual nature of motivation, that is implicit and explicit, the motivation process is complexity. In general, implicit motivational processes are primary and more essential than explicit motivational processes. Here we only focus on explicit motivation and hypothesize that the explicit motivational representations consist mainly of explicit goals of an agent. Explicit goals provide specific and tangible motivations for actions. Explicit goals also allow more behavioral flexibility and formation of expectancies. In cyborg intelligent system we have developed two approaches for brain computer integration, that is, needs based motivation and curiosity based motivation.

\subsection{Needs based motivation}

In 1943, humanistic psychologist Maslow put forward the demand theory of motivation. Maslow's assumption that people in need, the sequence of human motivation, from the most basic physiological and safety needs, through a series of love and respect, the complex needs of self-realization, and need level has great intuitive appeal [7]. Over the years, people have proposed a lot of theories of motivation, each theory has a certain degree of concern. These theories are very different in many ways, but they all come from a similar consideration, namely behavioral arousing, point to and keep, these three points are the core of any kind of motivation.

Bach proposed the MicroPsi architecture of motivated cognition based on situated agents [8]. MicroPsi explores the combination of a neuro-symbolic cognitive architecture with a model of autonomous, polytelic motivation. The needs of MicoPsi cognitive system fall into three groups: physiological needs, social needs and cognitive [12]. Physiological needs regulate the basic survival of the organism and reflect demands of the metabolism and physiological well-being. Social needs direct the behavior towards other individuals and groups. They are satisfied and frustrated by social signals and corresponding mental representations. Cognitive needs give rise to open-ended problem solving, skill-acquisition, exploration, play and creativity. Urges reflect various physiological, social and cognitive needs. Cognitive processes are modulated in response to the strength and urgency of the needs.

According to brain computer integration requirements, a motivation could be represented as a 3-tuples $\{N, G, I\}$, where $N$ means needs, $G$ is goal, $I$ means the motivation intensity [13]. There are three type of needs in the cyborg system:

a. Perception needs: Acquire environment information through vision, audition, touch, taste, smell. 
b. Adaptation needs: Adapt environment condition and optimize impaction of action.

c. Cooperation needs: Promise to reward a cooperation action between brain and machine.

A motivation is activated by motivational rules which structure has following format:

$$
\mathrm{R}=(P, D, \text { Strength }(P \mid D))
$$

where, $P$ indicates the conditions of rule activation; $D$ is a set of actions for the motivation; Strength $(P \mid D)$ is a value within interval $[0,1]$.

\subsection{Curiosity based motivation}

Curiosity based motivation is through motivation learning algorithm to build a new motivation. Agent creates internal representations of observed sensory inputs and links them to learned actions that are useful for its operation. If the result of the machine's action is not relevant to its current goal, no motivation learning takes place. This screening of what to learn is very useful since it protects machine's memory from storing unimportant observations, even though they are not predictable by the machine and may be of sufficient interest for novelty based learning. Novelty based learning still can take place in such a system, when the system is not triggered by other motivations.

Motivation learning requires a mechanism for creating abstract motivations and related goals. Once implemented, such a mechanism manages motivations, as well as selects and supervises execution of goals. Motivations emerge from interaction with the environment, and at any given stage of development, their operation is influenced by competing event and attention switching signals.

The learning process for motivations to obtain the sensory states by observing, then the sensed states are transformed mutually by the events. Where to find novelty to motivate an agent's interestingness will play an important role. Once the interestingness is stimulated, the agent's attention may be selected and focused on one aspect of the environment. Therefore, it will be necessary to define observations, events, novelty, interestingness and attention before descripting the motivation learning algorithm.

Definition 1: Observation Functions

Observation functions define the combinations of sensations from the sensed state that will motivate further reasoning. Observations containing fewer sensations affect an agent's attention focus by making it possible for the agent to restrict its attention to a subset of the state space. Where, a typical observation function can be given as:

$$
\mathbf{0}_{\mathrm{S}(\mathrm{t})}=\left\{\left(\mathrm{o}_{1(\mathrm{t})}, \mathrm{o}_{2(\mathrm{t})}, \cdots, \mathrm{o}_{\mathrm{L}(\mathrm{t})}, \cdots\right) \mid \mathrm{o}_{\mathrm{L}(\mathrm{t})}=\mathrm{s}_{\mathrm{L}(\mathrm{t})}(\forall \mathrm{L})\right\}
$$

The equation defines observation function $\mathbf{0}_{\mathrm{S}(\mathrm{t})}$ in which each observation focuses on every element of the sensed state at time $t$.

\section{Definition 2: Difference Function}

A difference function $\Delta$ assigns a value to the difference between two sensations $\mathrm{S}_{\mathrm{L}(\mathrm{t})}$ and $\mathrm{S}_{\mathrm{L}\left(\mathrm{t}^{\prime}\right)}$ in the sensed states $\mathrm{S}_{(\mathrm{t})}$ and $\mathrm{S}_{\left(\mathrm{t}^{\prime}\right)}$ as follows: 


$$
\Delta\left(\mathrm{s}_{\mathrm{L}(\mathrm{t})}, \mathrm{s}_{\mathrm{L}\left(\mathrm{t}^{\prime}\right)}\right)= \begin{cases}\mathrm{s}_{\mathrm{L}(\mathrm{t})} ; & \text { if } \neg \exists \mathrm{s}_{\mathrm{L}\left(\mathrm{t}^{\prime}\right)} \\ \mathrm{s}_{\mathrm{L}\left(\mathrm{t}^{\prime}\right) ;} & \text { if } \neg \exists \mathrm{s}_{\mathrm{L}(\mathrm{t})} \\ \mathrm{s}_{\mathrm{L}(\mathrm{t})}-\mathrm{s}_{\mathrm{L}\left(\mathrm{t}^{\prime}\right)} ; & \text { if } \mathrm{s}_{\mathrm{L}(\mathrm{t})}-\mathrm{s}_{\mathrm{L}\left(\mathrm{t}^{\prime}\right)} \neq 0 \\ 0 ; & \text { otherwise }\end{cases}
$$

Difference function offers the information about the change between successive sensations it calculates the magnitude of the change.

\section{Definition 3: Event Function}

Event functions define which combinations of difference variables an agent recognizes as events, each of which contains only one non-zero difference variable. Event function can be defined as following formula:

Where,

$$
\mathbf{E}_{\mathrm{S}(\mathrm{t})}=\left\{\mathrm{E}_{\mathrm{L}(\mathrm{t})}=\left(\mathrm{e}_{1(\mathrm{t})}, \mathrm{e}_{2(\mathrm{t})}, \cdots, \mathrm{e}_{\mathrm{L}(\mathrm{t})}, \cdots\right) \mid \mathrm{e}_{\mathrm{e}(\mathrm{t})}\right\}
$$

$$
\mathrm{e}_{\mathrm{e}(\mathrm{t})}= \begin{cases}\Delta\left(\mathrm{s}_{\mathrm{e}(\mathrm{t})}, \mathrm{s}_{\left.\mathrm{e}\left(\mathrm{t}^{\prime}\right)\right) ;}\right. & \text { if } \mathrm{e}=\mathrm{L} \\ 0 ; & \text { otherwise }\end{cases}
$$

Events may be of varying length or even empty, depending on the number of sensations to change.

\section{Definition 4: Novelty Detection Function}

The novelty detection function, $\mathbf{N}$, takes the conceptual state of the agent, $\mathbf{c} \in \mathbf{C}$, and compares it with memories of previous experiences, $m \in \mathbf{M}$, constructed by long term memory to produce a novelty state, $\mathrm{n} \in \mathbf{N}$ :

$$
\mathbf{N}: \mathbf{C} \times \mathbf{M} \rightarrow \mathbf{N}
$$

Novelty can be detected by introspective search comparing the current conceptual state of an agent with memories of previous experiences [14].

\section{Definition 5: Interestingness Function}

The interestingness function determines a value for the interestingness of a situation, $\mathrm{i} \in \mathbf{I}$, basing on the novelty detected, $\mathrm{n} \in \mathbf{N}$ :

$$
\text { I : } \mathbf{N} \rightarrow \mathbf{I}
$$

Definition 6: Attention Selection

Selective attention enables you to focus on an item while mentally identifying and distinguishing the non-relevant information. In cyborg we adopt maximal interestingness strategy to select attentions to create a motivation.

The following describes the basic steps of novelty based motivation learning and goal creation algorithm in the cyborg system.

Motivation learning algorithm

(1) Observe $\mathbf{0}_{\mathrm{S}(\mathrm{t})}$ from $\mathbf{S}_{(\mathrm{t})}$ using the observation function

(2) Subtract $\mathbf{S}_{(\mathrm{t})}-\mathbf{S}_{\left(\mathrm{t}^{\prime}\right)}$ using the difference function

(3) Compose $\mathbf{E}_{\mathrm{S}(\mathrm{t})}$ using the event function

(4) Look for $\mathbf{N}_{(t)}$ using introspective search

(5) Repeat (for each $\mathrm{N}_{\mathrm{i}}(\mathrm{t}) \in \mathbf{N}(\mathrm{t})$ )

(6) Repeat (for each $\mathrm{I}_{\mathrm{j}}(\mathrm{t}) \in \mathbf{I}(\mathrm{t})$ )

(7) Attention $=\max \mathrm{I}_{\mathrm{j}}(\mathrm{t})$

(8) Create a Motivation by Attention . 


\subsection{Motivation execution}

Motivation execution flow is shown in Figure 3. The awareness gets information from the environment and places it into the event list. Select one event from event list and identify it. If the event is a normal event then retrieval the motivation base and select one motivation to activate the intention. If the event is a new one and never happened previously, then call motivation learning to generate a new motivation. The new motivation will activate the intention. Based on the intention plan execution will be caused and generate a series actions to accomplish the desired goal, which requires the cooperation of the reasoning machine. This means that the system will find one or more of schemes which are made in the past. It is possible to find a solution that is not the only solution when it is used to reason about an existing object. At this time, the inference engine needs to select according to its internal rules. The selection criteria need to specify before. Different selection criteria will lead to the agent different behavioral responses at the decision-making. After choosing a good plan, the system will need to link up the goal and the plan in advance. This will make the planning a detailed understanding of the objectives, and there is sufficient information to be able to use for making planning of the goal.

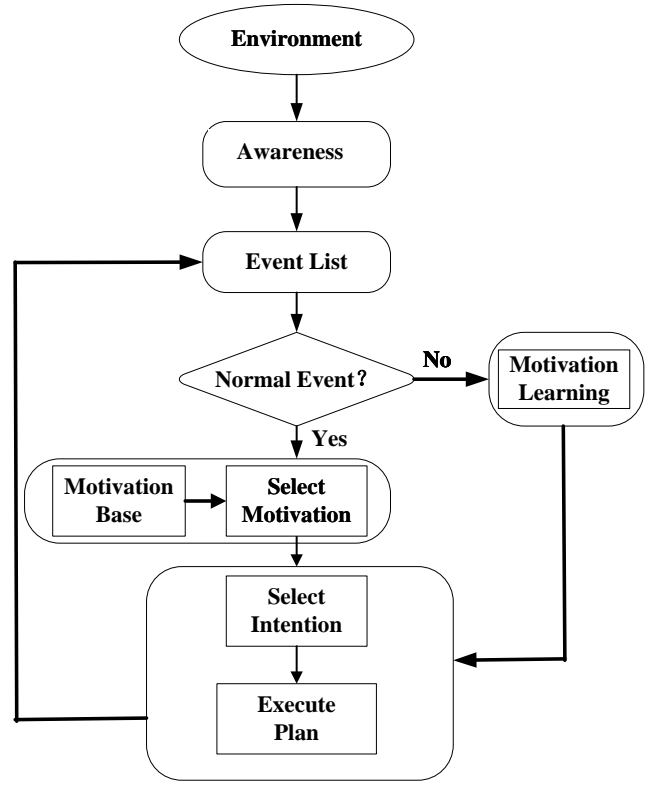

Fig. 3. Motivation execution

In cyborg system, the realization of the motivation module is through agent model ABGP. The current belief of the belief memory storage contains the agent motivation base. A desire is a goal or a desired final state. Intention is the need for the smart body to choose the current implementation of the goal. In agent, the goal is a directed acyclic graph by the sub goal composition, and realizes in step by step. According to a directed acyclic graph a sub goal is represented by a path to complete, the total goal will finish when all sub goals are completed. 


\subsection{Collaboration}

In brain-machine integration rat brain should work with machine collaboratively. Here rat brain and machine can be abstracted as agent, so the collaboration can be viewed as joint intention [14]. Joint intention is about what the team members want to achieve. Each team member knows the intention specifically and achieves it by collaboration.

In the joint intention theory, a team is defined as "a set of agents having a shared objective and a shared mental state". The team as a whole holds joint intentions, and each team member must inform others whenever it detects the goal state change, such as goal is achieved or the goal is no longer relevant.

For the joint intention, rat agent and machine agent have three basic knowledge: first, each one should select its intention; second, each one knows its cooperator who also select the same intention; and last, each one knows they are a team. They can know each other through agent communication.

\section{Conclusions}

This paper described cyborg intelligence which integrates the best of both machine and biological intelligences via brain-machine integration. To make this integration effective and co-adaptive biological brain and machine should work collaboratively. Both environment awareness based collaboration and motivation based collaboration are presented in the paper. The motivation leaning algorithm is explored in terms of event curiosity, which is useful for sharing common interest situations. Under situations appear repeatedly the motivation is selected by knowledge rules.

The future of cyborg intelligence may lead towards many promising applications, such as neural intervention, medical treatment, and early diagnosis of some neurological and psychiatric disorders. Cyborg intelligence has the potential to make the bionic man reality.

The cyborg intelligence is one approach to reach the human-level intelligence. A lot of basic issues of brain-like general intelligent systems are explored in the book [15] in detail.

\section{Acknowledgements}

This work is supported by the National Program on Key Basic Research Project (973) (No. 2013CB329502), National Natural Science Foundation of China (No. 61035003), National Science and Technology Support Program (2012BA107B02).

\section{References}

1. Clynes, M.E. and Kline, N.S.: Cyborgs and Space. Astronautics, pp. 26-27, 74-76, Sept. (1960)

2. Talwar, S.K., Xu, S., Hawley, E.S., Weiss, S.A., Moxon, K.A., and Chapin, J.K.: Behavioural neuroscience: Rat navigation guided by remote control. Nature, 417(6884):37-38 (2002)

3. Gray, B.: Collaborating: Finding Common Ground for Multiparty Problems. San Francisco: Jossey-Bass (1989)

4. Cohen, P.R. and Levesque, H. J.: Intention is choice with commitment. Artificial 
Intelligence 42(2-3):213-361 (1990)

5. Gutwin, C., Greenberg, S.: The Importance of Awareness for Team Cognition in Distributed Collaboration. Team Cognition: Understanding the Factors That Drive Process and Performance, E. Salas and S. Fiore, eds, 177-201 (2004)

6. Mook, D. G.: Motivation: The Organization of Action. W. W. Norton and Company, Inc, New York (1987)

7. Maslow, A.H.: Motivation and Personality. Boston: Addison-Wesley (1954, 1970,1987)

8. Bach, J.: Principles of Synthetic Intelligence - An architecture of motivated cognition. Oxford University Press (2009)

9. Zhaohui Wu, Gang Pan, José Carlos Príncipe, Andrzej Cichocki.: Cyborg Intelligence: Towards Bio-Machine Intelligent Systems. IEEE Intelligent Systems 29(6): 2-4 (2014)

10. Yipeng Yu, Nenggan Zheng, Zhaohui Wu, Xiaoxiang Zheng, Weidong Hua, Chen Zhang, Gang Pan: Automatic training of ratbot for navigation, International Workshop on Intelligence Science, in conjunction with IJCAI-2013, Beijing, China (2013)

11. Gang Ma, Xi Yang, Bo Zhang, Baoyuan Qi, Zhongzhi Shi: An Environment Visual Awareness Approach in Cognitive Model ABGP. 27th IEEE International Conference on Tools with Artificial Intelligence, November: 744-751 (2015)

12. Bach, J.: Modeling Motivation in MicroPsi 2. AGI 2015: 3-13 (2015)

13. Zhongzhi Shi, Jianhua Zhang, Jinpeng Yue, Baoyuan Qi: A Motivational System For Mind Model CAM. AAAI Symposium on Integrated Cognition, 79-86, Virginia, USA (2013)

14. Zhongzhi Shi, Jianhua Zhang, Xi Yang, Gang Ma, Baoyuan Qi, and Jinpeng Yue: Computational Cognitive Models for Brain-Machine Collaborations. IEEE Intelligent Systems, 11/12, 24-31 (2014)

15. Zhongzhi Shi: Mind Computation (in Chinese). Tsinghua University Press (2015) 